\title{
The Research on One Feasible Test Strategy for Temperature Rising Limits of Low-Voltage Metering Cabinets
}

\author{
SHEN Hua ${ }^{1}$, CHEN Sheng ${ }^{2}$, ZHANG Penghe ${ }^{2}$, Wang Feng ${ }^{1}$, ZHEN Haohan ${ }^{1}$ \\ ${ }^{1}$ State Grid Shanghai Electric Power Research Institute, Shanghai, China \\ ${ }^{2}$ China Electric Power Research Institute, Beijing, China
}

KEY WORDS : Low-voltage metering cabinet, Temperature rising limit, Grey model, Test strategy.

\begin{abstract}
The consumption of electric power is increasing with the continuous development of China's economy. The temperature rising limits testing is the important performance for low-voltage metering cabinets and circuit breakers. The strategy for temperature rising testing usually had many questions such as the style of thermocouple pasting, long testing time, the randomness by operator and so on. According to amount experiences for low-voltage metering cabinet, a good strategy for temperature rising limit testing was proposed in the paper. Meanwhile, the grey model used to analysis the temperature rising data for the growth trend of data itself.
\end{abstract}

\section{Background}

The consumption of electric power is increasing with the continuous development of China's economy. The safety and stability of residential electricity for the augment of household appliances. The low-voltage metering cabinets is the important line of defense to protect the operation of the electric meters, it also would be the foundation of the safety for electric meters and elements. The temperature rising limits testing is the important performance for low-voltage metering cabinets and circuit breakers. A good performance on the temperature rising limits could avoid to make some fire accidents caused by problems of wires.

The national standards GB 7251.1 and GB 10963 have given a clearly definition, the temperature rising limits of one component or part was difference between the measurements of temperature for the component or part and the air temperature of the external cabinets. Meanwhile, the section 9.8.3 said it should use a thermocouple or an equivalent tool to complete the measurement on the hottest point which could be close to. And also the thermocouple and the surface of the hottest point should be kept a good thermal conductivity. Both the different ways to paste and the choices of the measuring point would lead to some changes for the results of temperature rising limits measurement.

The low-voltage metering cabinets were usually as the basement used to install electric meters, voltage and current transformers, acquisition terminals and breakers. According to the rules of state grid standard Q/GDW 11008, the section area of wires should be $16 \mathrm{~mm}^{2}$ and the length of wires should be $1 \mathrm{~m}$ while the testing current was 60A. On the other hand, the section area of wires need $20 \mathrm{~mm}^{2}$ when the testing current was 100A. The qualifications of the temperature rising limits for different parts were showed in Table.1. 
Table.1. The qualifications of temperature rising limits for parts in low-voltage metering cabinets

\begin{tabular}{|c|c|c|}
\hline Number & The parts of low voltage metering cabinets & $\begin{array}{c}\text { Temperature } \\
\text { rising limits }(K)\end{array}$ \\
\hline 1. & $\begin{array}{l}\text { The Plug Module } \\
-----60 A \\
----100 A\end{array}$ & $\begin{array}{l}60 \\
70\end{array}$ \\
\hline 2. & $\begin{array}{l}\text { Circuit Breakers } \\
-----C B \\
----M C B\end{array}$ & $\begin{array}{l}70 \\
60\end{array}$ \\
\hline 3. & $\begin{array}{l}\text { Operating Handles } \\
\text {-----Metal } \\
\text {-----Nonmetal }\end{array}$ & $\begin{array}{l}15 \\
25\end{array}$ \\
\hline 4. & Shell & 30 \\
\hline
\end{tabular}

There are too many kinds of low-voltage metering cabinet in the market, and the quality of inner circuit breakers has the different level. All this questions lead a complex operation environment for the smart meters, so that easily happen some accidents such as the overheating and fire. It is necessary to do the temperature rising limits testing which could keep the low-voltage metering cabinet operating safety and stability. In the paper, a feasible testing strategy for temperature rising limits was proposed to simplify the existing testing methods based on the grey model.

\section{The principle of temperature rising}

As we know, the wires would emit heat when a fixed current across the line caused by the contact resistant which was usually called the heat effect of electric current. The formula about a connecting terminal was showed as follow.

$$
\tau_{a}=\frac{I^{2} \rho}{K_{r} p S}
$$

In the formula, $\tau_{a}$ was the temperature rising, $I$ was the current across the wires (A), $\rho$ was the resistivity of conductor $(\Omega / \mathrm{m}), K_{r}$ was the integrated surface heat transfer coefficient of conductor $\left(\mathrm{W} /\left(\mathrm{m}^{2} \bullet \mathrm{kj}\right)\right)$ and the value should be among 6 and 9 while the conductor used copper, $p$ was the circumference of cross section $(\mathrm{m})$ and $\mathrm{S}$ was the cross section $\left(\mathrm{m}^{2}\right)$.

The facts to effect the temperature rising mainly included the current, resistivity and diameter of conductors. The formula (1) could simplify to the function of conductor diameter when the conductor was cylinder with a tight connection. Meanwhile, both the circumference of cross section and cross section were the function of diameter. then could get the function between the temperature rising and conductor diameter as shown in formula (2).

$$
\tau_{a}=4 \times \frac{I^{2} \rho}{K_{r} \pi^{2} D^{3}}
$$

Taking the terminal of the electric meters as an example, if the current was $60 \mathrm{~A}$, the diameter of the terminal hole was $\Phi 7.5 \mathrm{~mm}$, the cross section of wires was $16 \mathrm{~mm}^{2}$, the integrated surface heat transfer coefficient of conductor was $8 \mathrm{~W} /\left(\mathrm{m}^{2} \cdot \mathrm{kj}\right)$, the resistivity of copper was $1.7 \times 10^{-8} \Omega / \mathrm{m}$, the 
result of theory temperature rising limits was just $25.86 \mathrm{~K}$. But there were many facts as like the installation and connection in actual operation, the actual temperature rising would greatly exceed the theory value by the larger contact resistance. The width of electric connection terminals was 24 $\mathrm{mm}$ and the thickness was $5 \mathrm{~mm}$, and then could calculate the theory temperature rising limits was $38.25 \mathrm{~K}$ according to the formula (1) if the contact area was about $20 \mathrm{~mm}^{2}$ with the wires.

3 The temperature rising limit testing

According to amount experiences for low-voltage metering cabinet, a good strategy for temperature rising limit testing was proposed in the paper which included the ready works for electric connection, the temperature measurement by use of infrared detector, the style of thermocouple pasting, the real-time monitoring for temperature rising and data analysis.

(1) The ready works for electric connection

The wires for low-voltage metering cabinet used the reverse connection, the lines from the current source were accessing to the breakers and used a short circuit for switches. For example, the electric connection method for a single phase low-voltage metering cabinet was showed in Fig.1.

(2) The temperature measurement by use of infrared detector

The infrared detector should be needed to ensure the hottest position when tested a new type of samples, it could intuitively describe the different temperature area through the photographs as shown in Fig.2.

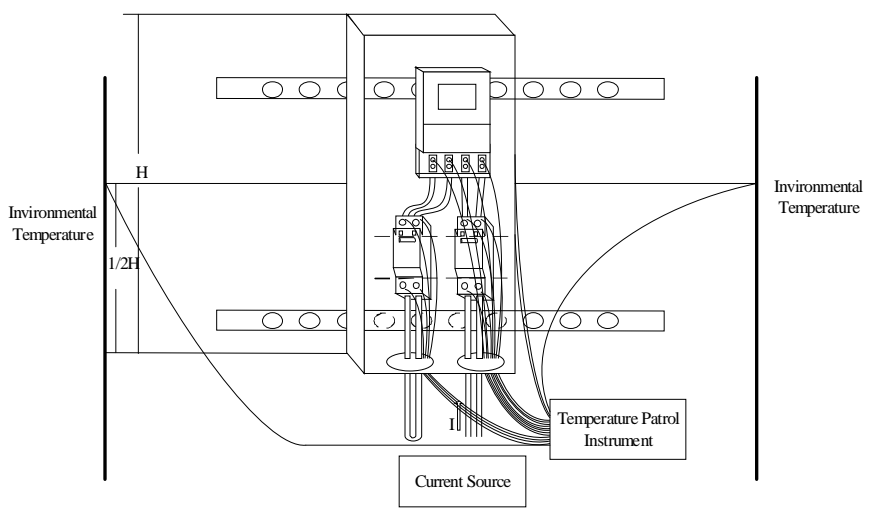

Figure.1. The electric connection method for a single phase temperature low-voltage metering cabinet detector

(3) The style of thermocouple pasting

The temperature rising limit should be accurate and closed to the hottest temperature, it is necessary to use the contact temperature measurement. The Satlon adhesives and Satlon catalysts were used to complete the thermocouple pasting. Firstly a little adhesive would be taken on thermocouple, and then quickly put the thermocouple on the surface of the test point and kept a tight connection, finally used catalysts to cool the adhesive, further to complete the pasting.

(4) The real-time monitoring for temperature rising

The patrol instrument was used to monitor the temperature in real-time, and the testing should be ended when ensure the change of temperature not exceed $1 \mathrm{~K}$ per hour by using the statistical method. The "Pass" result should be given if all the temperature rising limit data were inner qualified range, otherwise, gave the "Fail" result.

(5) Data analysis

The whole temperature real-time monitoring data should be saved as the attachment for original record of temperature rising limit testing. 
The temperature rising limit data for breakers in low-voltage metering cabinet could be recorded by the step (1) to (5) as shown in Figure.3. It was easy to know the temperature rising data had an obviously growth trend at the beginning stage.

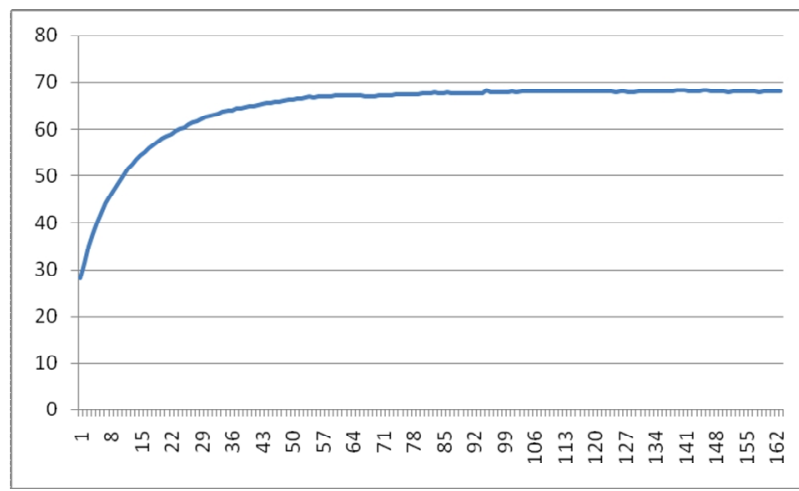

(a) Point one

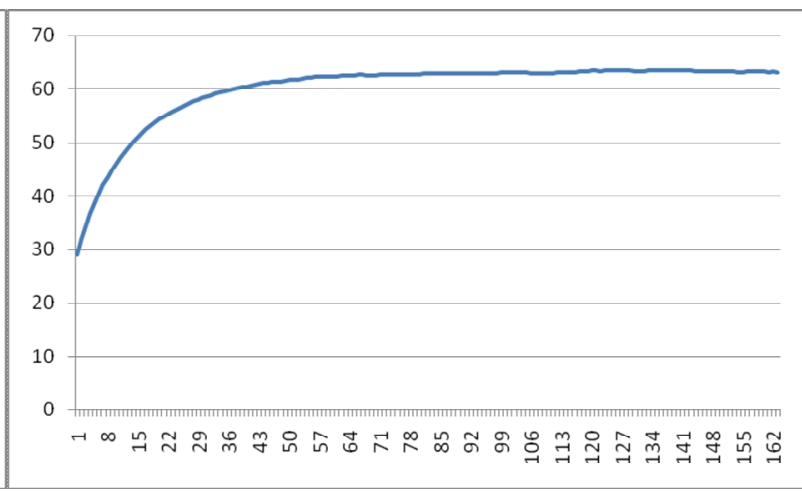

(b) Point two

Figure. 3 The temperature rising limit data of measurement point one

\section{Modeling in Grey Model}

The grey model should be used to analysis the temperature rising data for the growth trend of data itself. The GM $(1,1)$ model was the most representative in grey models which consisted of only one order differential equation with one variable. The model GM $(1,1)$ could be an effective model for prediction as the special model of GM $(1, n)$. The detail steps to model the GM $(1,1)$ as follow.

Set the original data of temperature rising for $\mathrm{k}^{(0)}$ sequence as formula (3).

$$
k^{(0)}=\left[k^{(0)}(1), k^{(0)}(2), \mathrm{L}, k^{(0)}(n)\right]
$$

Generated the sequence of first order accumulation as formula (4).

$$
k^{(1)}=\left[k^{(1)}(1), k^{(1)}(2), \mathrm{L}, k^{(1)}(n)\right]
$$

And

$$
k^{(1)}(m)=\sum_{i=1}^{m} k^{(0)}(i) \quad(\mathrm{m}=1,2, \ldots, \mathrm{n})
$$

Because the $k^{(1)}(\mathrm{m})$ sequence had exponential growth trend and the first order differential equation could be used to solve the answer for exponential growth sequence. So it could be considered that $k^{(1)}$ sequence were suitable for the first order differential equation as formula (6).

$$
\frac{d k^{(1)}}{d t}+a \cdot v=u
$$

It was necessary to solve the value of parameter $a$ and $u$ which represented the development coefficient and control parameter before solving the differential equation. According to the definition of the derivative, the formula (6) could be got as follow.

$$
\frac{d k^{(1)}}{d t}=\lim _{\Delta \mathrm{t} \rightarrow 0} \frac{k^{(1)}(t+\Delta \mathrm{t})-k^{(1)}(t)}{\Delta \mathrm{t}}
$$

The differential term could describe as formula (8) in discrete form. 


$$
\begin{aligned}
\frac{\Delta k^{(1)}}{\Delta t} & =\frac{k^{(1)}(m+1)-k^{(1)}(m)}{m+1-m} \\
& =k^{(1)}(m+1)-v^{(1)}(m) \\
& =k^{(1)}\left[k^{(1)}(m+1)\right] \\
& =k^{(0)}(m+1)
\end{aligned}
$$

And the $k^{(1)}$ was the mean value between the time $\mathrm{m}$ and time $(\mathrm{m}+1)$ as shown in formula (9).

$$
\frac{1}{2}\left[k^{(1)}(m+1)+k^{(1)}(m)\right]
$$

Therefore, the differential equation could change to be the equation (10).

$$
a^{(1)}\left[k^{(1)}(m+1)\right]+\frac{1}{2}\left[k^{(1)}(m+1)+k^{(1)}(m)\right]=u
$$

The result of equation (9) should be formula (10) by writing the results above in matrix line when the variable $m$ change with the number.

$$
\left[\begin{array}{c}
k^{(0)}(2) \\
k^{(0)}(3) \\
\mathrm{M} \\
k^{(0)}(n)
\end{array}\right]=\left(\begin{array}{cc}
-\frac{1}{2}\left[k^{(1)}(1)+k^{(1)}(2)\right] & 1 \\
-\frac{1}{2}\left[k^{(1)}(2)+k^{(1)}(3)\right] & 1 \\
\mathrm{M} & \mathrm{M} \\
-\frac{1}{2}\left[k^{(1)}(n-1)+k^{(1)}(n)\right] & 1
\end{array}\right)\left(\begin{array}{l}
a \\
u
\end{array}\right)
$$

Because of the variables were only $a$ and $u$ in above equations, meanwhile the number of equations had (n-1) and (n-1) exceed two, the equations had no result. Finally the least square method could be used to obtain the least square approximation. Took the parameters $a$ and $u$ into differential equation (1), it could solve the sequence $\mathrm{k}^{(1)}$ as formula (12).

$$
k^{(1)}(t+1)=\left[k^{(1)}(1)-\frac{\bar{u}}{\bar{a}}\right] e^{-\bar{a} t}+\frac{\bar{u}}{\bar{a}}
$$

Make the regressive reduction for equation (12), and then got the original temperature rising sequence $\mathrm{k}^{(0)}$ as shown in formula (13).

$$
k^{(0)}(k+1)=k^{(1)}(m+1)-k^{(1)}(m)=\left(1-e^{\bar{a}}\right)\left[v^{(0)}(1)-\frac{\bar{u}}{\bar{a}}\right] e^{-\bar{a} m} \quad(\mathrm{~m}=0,1,2 \ldots)
$$

Chose the temperature rising data which had been showed in Fig.3 to get a suitable model based on formula (10). The grey model was showed in formula (14) with the parameter $a$ was -0.115 and the parameter $u$ was 36.39 . It was significance that the grey model could use a shorter time to predict the finally testing data. However, more works and researches should do in the future to solve the accuracy of the model.

$$
\begin{aligned}
k^{(0)}(m+1) & =k^{(1)}(m+1)-k^{(1)}(m)=\left(1-e^{\bar{a}}\right)\left[k^{(0)}(1)-\frac{\bar{u}}{\bar{a}}\right] e^{-\bar{a} m} \\
& =\left(1-e^{-0.115}\right) \times\left(28.2-\left(\frac{36.39}{-0.115}\right)\right) \times e^{-0.115 m}
\end{aligned}
$$

\section{Conclusion}

The grey model firstly used to analysis the temperature rising data for the growth trend of data itself in the paper. It mainly hoped to solve the testing problems such as the long testing term and 
accuracy of results. The next work should look for the corresponding relation between the parameters $\mathrm{a}, \mathrm{u}$ and the physical variables included current, diameter of wires, contact resistance and so on.

\section{Reference}

[1] GB 7251.1-2013 Low-voltage switchgear and controlgear assemblies- Part 1: General rules [S].

[2] GB 10963.1-2006 Electrical accessories- Circuit - breakers for overcurrent protection for household and similar installation- Part 1: Circuit-breakers for a.c. operation [S].

[3] Liu Gang, Lei Chenghua, Analysis on transient error of simplified thermal circuit model for calculating conductor temperature by cable surface temperature [J], Power System Technology, 2011, 35(4): 212-217

[4] Liu Yigang, Luo Junhua, Mathematical method of temperature calculation of power cable conductor in real time [J], High Voltage Engineering, 2005, 31(5): 52-54

[5] DengJulong. Application of grey system theory in China. In: First international symposium on uncertainty modeling and analysis December 1990.p.285-291

[6] Ruiqing Wang, Lian Yao, Yuzeng Li. A hybrid forecasting method for day-ahead electricity price based on GM $(1,1)$ and ARMA. In: IEEE International Conference on Grey Systems and Intelligent Services November 2009.p.577-581

[7] CHEN Sheng, YE Lin, Short-term wind power Prediction based on Combined Grey-Markov Model. In: 2011APAP International Conference on Beijing, July 2011. 